\title{
Problemas de salud y patrones de comportamiento
}

- José Luis Henriquez

\section{Introducción}

Existe un tipo de problemas de salud en los que lo psicológico constituye el primer eslabón interno en su determinación. A tales problemas se les ha aplicado, tradicionalmente, el término de enfermedades psicosomáticas.

Los trastomos psicosomáticos o psicofisiológicos son enfermedades físicas que incluyen varios grupos de condiciones marcadas por el daño real del tejido orgánico o su deterioro, a las que se les atribuye un origen o un componente predominantemente psicológico (Nathan, P. E. y Harris, S.L. Psicopatología y Sociedad. $2^{\mathrm{i}}$. Edición. Editorial Trillas, México 1989.), ya que los síntomas corporales se relacionan íntimamente con ciertos factores de la personalidad y el entorno y/o con los sucesos que tienen una importancia psicológica. (Sarason, I.G. y Sarason, B.R. Psicología Anormal: El problema de la conducta inadaptada. $7^{\mathrm{a}}$ Edición. Editorial Prentice-Hall, México, 1996.)

Es oportuno mencionar que a estos problemas de salud se les denomina oficialmente como "factores psicológicos y del comportamiento asociados con trastornos o enfermedades clasificadas en otra parte"; la Clasificación Internacional de Enfermedades (Organización Mundial de la Salud (OMS). CIE 10 / Trastornos mentales y del comportamiento: Descripciones Clínicas y Pautas para el Diagnóstico. Editorial Meditor, Madrid, 1992.), designa con esta expresión a la categoría (F54) que registra la presencia de factores psicológicos o conducluales, que se considera han desempeñado un papel principal en la etiología de trastornos físicos que pueden clasificarse en sus correspondientes apartados.

Problemas de salud y patrones de comportariento 
La característica de este grupo de trastomos es que los síntomas físicos corresponden a un solo șistema orgánico, generalmente bajo la influencia del sistema nervioso autónomo (Nathan, P.E. y Harris, S. L. Op. cit.); entre los que se comprenden los sistemas cardiovascular, respiratorio, gastrointestinal, musculoesquelético, genitourinario, así como la piel (Sarason, I.G. y Sarason, B.R. Op. cit.).

Se reconoce que la diferenciación entre los trastornos psicosomáticos y los trastomos orgánicos que son similares - hay que tener en cuenta que los primeros también pueden tener una etiología predominantemente física-, es usualmente difícil, pues no existen indicadores definidos que gocen de consenso para establecer tal distinción( Nathan, P.E. y Harris, S. L. Op. cit.).

Debe aceptarse también que la falta de acuerdo se extiende al ámbito de la explicación del mecanismo a través del cual el o los factores etiológicos dan origen al trastomo.

Se ha especulado que un factor psicológico específico determina un trastomo psicosomático específico. Por ejemplo, se han presumido asociaciones entre dependencia y úlcera, competitividad hostil e hipertensión, agresividad y migraña (este punto de vista se ha conocido como modelo de la especificidad) (Ídem).

Un planteamiento con suficiente apoyo empírico considera que cada individuo reacciona con respuestas autónomas características y consistentes ante cualquier estímulo emocional. Por ejemplo, algunos responden vigorosamente con cambios en la frecuencia cardíaca, la presión arterial o la motilidad gástrica, siendo ello lo que determina un trastomo psicosomático específico (esta hipótesis se conoce como modelo de la especificidad de la respuesta individual). (Ídem) La consistencia de tal patrón fisiológico, a lo largo de la vida del individuo, identificado incluso en recién nacidos, señala la implicación de un factor constitucional. (Rosenzweig, M.R. y Leiman, A. I. Psicología fisiológica. 2ª Edición. Editorial McGraw-Hill, México, 1995)

En un sentido más particular, se ha argumentado que las respuestas emocionales se reflejan en los cambios del funcionamiento autónomo, al persistir la respuesta se altera la regulación de las funciones de la división simpática y parasimpática, dejando al organismo desprovisto de un sistema de inspección y balanceo, y es cuando puede haber un daño permanente del cuerpo (Nathan, P.E. y Harris, S.L. Op. cit.); los trastornos psicosomáticos se consideran, pues, un resultado de la estimulación fisiológica frecuente, intensa y prolongada.(Sarason, I.G. y Sarason, B.R. Op. cit.)

Se ha señalado, incluso, que hay un vínculo estrecho entre el tipo de emoción y la actividad de los órganos viscerales controlados por el sistema nervioso autónomo, por ejemplo, el incremento en la frecuencia del pulso y en la presión sanguínea es mayor en el miedo que en la cólera (Rosenzweig, M. R. y Leiman, A. I. Op. cit.); hallazgo que bien podría llevar a suponer que es el responder 
persistentemente con un determinado tipo de emoción, lo que da lugar a un determinado trastomo psicosomático.

Una línea de investigación, aún más circunscrita, es la relativa al análisis de la asociación entre el estilo de vida y uno de los trastornos del sistema cardiovascular: la enfermedad cardíaca coronaria o enfermedad coronaria del corazón (ECC).

Friedman y Rosenman, luego de estudiar múltiples casos de ataques cardíacos, concluyeron, en 1974, que hay, definitivamente, personas con un riesgo elevado de sufrirlos; esta propensión está asociada a aspectos básicos de la personalidad, los cuales se han designado bajo las denominaciones de conducta o personalidad tipo $A$. La personalidad tipo A constituye un patrón de comportamiento, relativamente estable, configurado por la combinación de impaciencia, competitividad, enojo y hostilidad; el patrón de comportamiento opuesto, se conoce como personalidad tipo B. Las personas con personalidad tipo A viven de una forma frenética y agitada, y tienden a realizar sus actividades bajo gran presión y a ser exigentes, cínicas, desconfiadas y hostiles en exceso. (Ídem); (Sarason, I.G. y Sarason, B.R. Op. cit.); (Sue, D., Sue, D. y Sue, S., Comportamiento anormal. 4a Edición Editorial McGraw-Hill, México, 1996)

Rosenman y otros demostraron en 1975 que los individuos con conducta tipo A presentan más del doble de enfermedades cardíacas que los individuos con conducta tipo B (Rosenzweig, M. R. y Leiman, A. I. Op. cit.); (Sarason, I.G. y Sarason, B.R. Op. cit.).

Haynes y otros establecieron, en 1980, que las mujeres tipo A, entre los 45 y 64 años, sufren casi dos veces más de ECC que las mujeres tipo B, si son trabajadoras, y casi 3 veces más, si son amas de casa; los hombres tipo A, entre los 45 y 74 años, sufren casi 3 veces más que los hombres tipo B, entre los empleados de oficina; pero no se presentan diferencias entre los obreros. (Ídem)

Miller y sus colaboradores encontraron, en 1991, que "entre hombres de mediana edad con enfermedad cardiaca coronaria, el 70\% fueron tipo A; en hombres saludables sólo el $46 \%$ fueron tipo A". (Sue, D., Sue, D. y Sue, S. Op. cit.; pág. 230)

Diferentes estudios, realizados durante la segunda mitad de la década de los ochenta y la primera mitad de la década de los noventa, coinciden al aportar evidencias de que la hostilidad es el factor significativo, de la personalidad tipo A, en la elevación del riesgo a sufrir una enfermedad coronaria. (Ídem); (Sarason, I.G. y Sarason, B.R. Op. cit.)

Ahora bien, a pesar de lo que revela esta información, sería erróneo concluir que la personalidad tipo A constituye una cualidad intrínsecamente anómala; existe la consideración de que ésta incluye factores que pueden ser benignos como también poner en riesgo a los individuos, (Sue, D., Sue, D. y Sue, S. Op. cit) y de hecho la mayoría de individuos tipo A no presentan patología coronaria; 
no obstante, la incidencia es superior a la que se produce en personas de tipo B (Rosenzweig, M. R. y Leiman, A. I. Op. cit.).

Pero, finalmente, todo esto constituye un conjunto de explicaciones parciales, relativamente independientes entre sí, que no proveen una explicación integral de los trastornos psicosomáticos.

De hecho, los mecanismos exactos que dan origen a estos trastomos siguen sin aclararse; sin embargo, cualesquiera que resulten ser, seguramente implican interacciones entre múltiples factores: personales (actitudes y patrones fisiológicos) y diversas situaciones (experiencias pasadas y recientes y apoyo social) (Sarason, I.G.

y Sarason, B.R. Op. cit .); esta noción se retomará más adelante.

El estudio que es objeto de este reporte, afín a la última línea de investigación mencionada, constituye un análisis de la asociación entre el patrón de comportamiento tipo A y diferentes trastornos psicosomáticos.

Su propósito es establecer la prevalencia, tanto de dicho patrón de comportamiento como de los problemas de salud en cuestión, en una detenninada población, así como comprobar la relación que hay entre ambos; con el objeto de brindar un aporte a la comprensión del papel de lo psicológico en la salud y la enfermedad.

La suposición, de la cual parte este examen, es que la personalidad tipo A no sólo favorece la enfermedad cardiaca, como ya se ha reseñado antes, sino también otros problemas de salud.

\section{Metodología}

Se hizo uso de un diseño epidemiológico de prevalencia o cruzado, diseño metodológico de tipo analítico no experimental, por lo que deben tenerse presentes las limitaciones en la generalización que pueda hacerse de los resultados obtenidos.

\subsection{Muestra}

La tabla 1 (ver anexo 1), presenta la distribución de la muestra, en diferentes variables demográficas.

Participaron en el estudio, un total de 300 estudiantes universitarios salvadoreños, a quienes se les consultó durante el mes de junio de 1997.

La muestra se compone de estudiantes que provienen proporcionalmente de las tres facultades de la Universidad Centroamericana "José Simeón Cañas" (33.7\% de Ciencias del Hombre y la Naturaleza, $33.3 \%$ de Ingeniería y $33.0 \%$ de Ciencias Económicas).

Las edades de los participantes están dispersas entre los 17 y los 50 años, por lo que se establecieron grupos de edad: 17-20 años $=32.3 \%$ (Jóvenes), $21-40$ años 
$=57.3 \%$ (Adultos jóvenes) y 41-50 años = 1.3\% (Adultos mayores); el $9.0 \%$ no respondió. La edad promedio del conjunto fue de 23 años.

En cuanto al sexo, las proporciones son similares. $45.7 \%$ eran mujeres y $53.7 \%$ hombres; el $0.7 \%$ no lo indicó.

En lo relativo al estado civil de los estudiantes participantes, la variación fue bastante alta: el $89.7 \%$ es soltero, el $6.7 \%$ casado o acompañado, el $0.7 \%$ divorciado o separado y el $0.7 \%$ viudo; el $2.3 \%$ no se estableció.

\subsection{Instrumentos}

El cuestionario Problemas de salud y patrones de comportamiento diseñado ad hoc, fue utilizado para realizar este estudio (ver anexo 2); el instrumento permite identificar y evaluar respectivamente: 1) Los problemas de salud padecidos y 2) Los rasgos característicos del tipo de personalidad.

La primera parte (ítems 1-3) recoge información demográfica: sexo, edad y estado civil.

La segunda parte (items 4-6) recoge información sobre problemas de salud padecidos (migraña, hipertensión, enfermedad del corazón, alergias o eccema, asma, úlcera, gastritis y colitis), las respuestas a estos 8 reactivos se codifican: $0=$ no se padece o $1=$ se padece o se ha padecido y, en este último caso, se registra el tiempo durante el cual ello ha ocurrido. También se consigna la experiencia de diversas situaciones de tensión/estrés y la opinión acerca de la relación existente entre éstas y los problemas de salud padecidos.

La tercera parte (ítem 7) consulta sobre el período de presencia de ciertos estados emocionales (intranquilidad, tristeza, temores y fatiga) y las respuestas se codifican: $0=$ períodos de horas o días, $1=$ períodos breves $(1$ mes $)$ y $2=$ períodos prolongados (más de 3 meses)

La cuarta parte (ítem 8) fue diseñada para evaluar 4 rasgos comportamentales: impaciencia, competitividad, enojo y hostilidad; esta medida consta de 23 reactivos $(8,4,8$ y 3 en cada rasgo respectivamente). Cada pregunta se codifica según el grado en que se reconoce como propio un determinado comportamiento y el margen de respuesta es de: $0=$ no me describe en absoluto, 1 = me describe en parte y 2 = me describe en gran medida; salvo en el caso de los últimos tres que se codifican de acuerdo a la frecuencia con que ocurre una conducta específica que va de $0=$ nunca, 1 = algunas veces, 2 = con frecuencia y $3=$ siempre. De los 23 reactivos formulados, se obtuvo una puntuación para cada uno de los factores y una puntuación total, la cual llevó a establecer los patrones de comportamiento o personalidad: tipo A, tipo B e intermedio entre los tipos A y B; tomando como criterio los puntajes por encima del percentil 75 , por debajo del percentil 25 y entre ambos respectivamente (ver anexo 3 ). 
El coeficiente alfa de la escala fue de 0.79 , los cuatro factores que resultan de esta escala fueron: impaciencia $(r=0.76)$, competitividad $(r=0.65)$, enojo $(r$ $=0.82)$ y hostilidad $(r=0.69)$

\subsection{Procedimiento}

Se solicitó información en la Secretaría General de la Universidad sobre las materias que estaban siendo impartidas, durante el período académico que correspondía al primer ciclo del año 1997, y el número de alumnos que estaban inscritos en ellas (exceptuando materias de maestrías y doctorados).

Luego, se eligió aleatoriamente una materia correspondiente a cada nivel de estudio de (Primero a quinto año), en cada una de las tres facultades (Quince materias en total), independientemente de la carrera a la que pertenecieran.

Finalmente, en cada una de las quince materias mencionadas, previamente autorizados por el profesor a cargo, se eligieron al azar veinte de los estudiantes que se encontraban presentes, a los cuales se les pidió responder el cuestionario.

Estas actividades fueron llevadas a cabo por un grupo de veintiséis estudiantes de psicología, que cursaban la materia de psicopatología de adultos.

\section{Resultados}

La tabla 2 (ver anexo 4) indica las frecuencias y proporciones de los tipos de personalidad identificados en las personas consultadas.

El $28.3 \%$ posee los rasgos característicos de la personalidad tipo A, el 27.7 $\%$ de la personalidad tipo $\mathrm{B}$ y el $44.0 \%$ de un patrón de comportamiento intermedio entre las personalidades tipo A y B; para efectos del estudio, estos dos últimos serán denominados tipo no $\mathrm{A}$.

La tabla 3 (ver anexo 5), presenta las frecuencias y proporciones de personas que refieren diferentes problemas de salud.

El $30.0 \%$ padece gastritis; el $27.7 \%$, alergias; el $16.7 \%$, migraña; el 11.3 $\%$, colitis; el $8.7 \%$, asma; el $6.3 \%$, alguna enfermedad cardíaca; el $5.3 \%$, hipertensión y el $3.7 \%$, úlcera.

Al hacer un análisis de estas frecuencias a través de tabulaciones cruzadas, se observa que no todas las personas consultadas (tipo A y tipo no A) padecen en igual medida otros problemas de salud.

La ocurrencia de casos de enfermedad cardiaca es superior entre las personas con rasgos de personalidad tipo A, con relación a quienes carecen de ellos, únicamente en el caso de personas de sexo femenino $\left(\mathrm{X}^{2}=4.85 ; \mathrm{p}<0.01\right)$, la fuerza de la relación, sin embargo, entre la característica y la condición, es débil $(C=0.19)$; entre los hombres no se constataron diferencias significativas $\left(X^{2}=\right.$ $0.18 ; \mathrm{p}<0.67)$ (ver cuadro 1 ). 


\section{Cuadro 1}

Relación entre la personalidad y el padecimiento de enfermedad cardíaca según sexo.

Padecimiento de enfermedad cardiaca

\begin{tabular}{|c|c|c|c|c|c|}
\hline & \multicolumn{2}{|c|}{ Femenino* } & \multicolumn{2}{|c|}{ Masculino** } & \\
\hline Personalidad & Sí & No & Sí & No & \\
\hline Tipo A & $613.6 \%$ & $\begin{array}{l}38 \\
86.4 \%\end{array}$ & $24.9 \%$ & $\begin{array}{l}39 \\
95.1 \%\end{array}$ & 85 \\
\hline Tipo no A & $\begin{array}{ll}3 & \\
& 3.2 \%\end{array}$ & $\begin{array}{l}9() \\
96.8 \%\end{array}$ & $8.7 \%$ & $\begin{array}{l}112 \\
93.3 \%\end{array}$ & 213 \\
\hline & 9 & $\begin{array}{l}128 \\
(137)\end{array}$ & 10) & $\begin{array}{l}151 \\
(161)\end{array}$ & $(298)$ \\
\hline
\end{tabular}

* $X^{2}=4.85(p<0.05) ; C=0.19 . \quad R R=4.7$

$* * X^{2}=0.18(p<0.67) ; C=0.03 . \quad R R=0.7$

En lo relativo al riesgo de sufrir enfermedad cardiaca, se estima que es aproximadamente cinco veces más probable entre las mujeres de personalidad tipo A $(\mathrm{RR}=4.7$, el cual constituye un riesgo elevado), respecto a las mujeres que les caracteriza otro tipo de personalidad.

La presencia de casos de migraña, igualmente, es significativamente mayor entre las personas con rasgos de personalidad tipo A, en comparación a las que no los poseen específicamente cuando se trata de personas de sexo femenino ( $\mathrm{X}^{2}$ $=8.47 ; \mathrm{p}<0.01)$, la fuerza de la relación, entre tal característica y tal condición, es levemente moderada $(C=0.24)$; entre los hombres no se encontraron diferencias significativas $\left(\mathrm{X}^{2}=0 .() 1 ; \mathrm{p}<0.94\right)($ ver cuadro 2$)$

\section{Cuadro 2}

Relación entre la personalidad y el padecimiento migraña, según sexo Padecimiento de migraña

\begin{tabular}{|c|c|c|c|c|}
\hline & \multicolumn{2}{|c|}{ Femenino* } & \multicolumn{2}{|c|}{ Masculino** } \\
\hline Personalidad & $\mathrm{Si}$ & No & $\mathbf{S i}$ & No \\
\hline Tipo A & $\begin{array}{l}15 \\
34.1 \%\end{array}$ & $\begin{array}{l}29 \\
65.9 \%\end{array}$ & $614.6 \%$ & $\begin{array}{l}35 \\
\quad 85.4 \%\end{array}$ \\
\hline Tipo no A & $\begin{array}{l}12 \\
12.9 \%\end{array}$ & $\begin{array}{l}81 \\
87.1 \%\end{array}$ & $\begin{array}{l}17 \\
14.2 \%\end{array}$ & $\begin{array}{l}1(1) 3 \\
\quad 85.8 \%\end{array}$ \\
\hline & 27 & $\begin{array}{l}110 \\
(137)\end{array}$ & 23 & $\begin{array}{l}138 \\
\quad(161)\end{array}$ \\
\hline
\end{tabular}

* $X^{2}=8.47(p<0.01) ; C=0.24 . R R=3.5$

$* * X^{2}=0.01(p<(0.94) ; C=0.01 . \quad R R=1.0$

Problemas de salud y pationes de comportamiento 
En lo referente al riesgo de sufrir migraña, se estima que es aproximadamente 4 veces más probable entre las mujeres de personalidad tipo $A(R R=3.5$, el riesgo es elevado), que entre las mujeres que no les caracteriza este tipo de personalidad.

Las personas con rasgos de personalidad tipo A, también, son las más afectadas por alergias, respecto aquellos sin estos rasgos, exclusivamente si éstas son de sexo masculino $\left(X^{2}=8.80 ; p<0.01\right)$, la fuerza de la relación, entre dicho tipo de personalidad y el problema de salud en cuestión, es ligeramente moderado (C $=0.23)$; entre las mujeres no se hallaron diferencias significativas $\left(X^{2}=0.47 ; p\right.$ < 0.49) (ver cuadro 3).

En lo relacionado al riesgo de padecer alergias, se estima que es aproximadamente tres veces más probable en los hombres de personalidad tipo $\mathrm{A}$ ( $\mathrm{RR}=$ 3.2 , el riesgo es elevado), que en los hombres que no poseen este tipo de personalidad.

No se encontraron diferencias significativas, entre las personas con personalidad tipo $\mathrm{A}$ y aquellos que no poseen este patrón de comportamiento, con respecto al padecimiento de gastritis $\left(X^{2}=0.49 ; p<0.48\right)$, colitis $\left(X^{2}=1.85 ; p<\right.$ $0.17)$, asma $\left(X^{2}=3.10 ; p<0.08\right)$, hipertensión $\left(X^{2}=0.30 ; p<0.58\right)$ y úlcera $\left(X^{2}=0.07 ; p<0.79\right)$.

\section{Cuadro 3}

Relación entre la personalidad y el padecimiento de alergias, según sexo Padecimiento de alergias

\begin{tabular}{|c|c|c|c|c|c|}
\hline \multirow[b]{2}{*}{ Personalidad } & \multicolumn{2}{|c|}{ Femenino* } & \multicolumn{2}{|c|}{ Masculino** } & \\
\hline & Si & No & $\mathbf{S i}$ & No & \\
\hline Tipo A & $\begin{array}{l}13 \\
29.5 \%\end{array}$ & $\begin{array}{l}31 \\
70.5 \%\end{array}$ & $\begin{array}{l}16 \\
39.0 \%\end{array}$ & $\begin{array}{l}25 \\
\quad 61.0 \%\end{array}$ & 85 \\
\hline \multirow[t]{2}{*}{ Tipo no A } & $\begin{array}{l}33 \\
35.5 \%\end{array}$ & $\begin{array}{l}60 \\
\quad 64.5 \%\end{array}$ & $\begin{array}{l}20 \\
\quad 16.7 \%\end{array}$ & $\begin{array}{l}100 \\
83.3 \%\end{array}$ & 21 \\
\hline & 46 & $\begin{array}{l}91 \\
(137)\end{array}$ & 36 & $\begin{array}{l}125 \\
(161)\end{array}$ & $(298)$ \\
\hline
\end{tabular}

* $\mathrm{X}^{2}=0.47(\mathrm{p}<0.49) ; \mathrm{C}=0.06 . \quad \mathrm{RR}=0.8$

** $\mathrm{X}^{2}=8.80(\mathrm{p}<0.01) ; \mathrm{C}=0.23 . \quad \mathrm{RR}=3.2$

En cuanto a las experiencias de situaciones provocadoras de tensión, se encontró que las personas con personalidad tipo A han estado mayormente expuestas a conflictos intrafamiliares $\left(X^{2}=19.46 ; p<0.01\right)$ en comparación a las 
personas sin este tipo de personalidad, el $71 \%$ de los primeros frente al $42 \%$ de los segundos; no se encontraron diferencias significativas, entre ambos grupos de personas, con respecto a su exposición a enfermedad de parientes $\left(X^{2}=2.85\right.$; $p<0.09)$, fallecimiento de seres queridos $\left(X^{2}=2.57 ; p<0.11\right)$, abuso sexual $\left(X^{2}\right.$ $=0.89 ; p<0.35)$, problemas con la ley $\left(X^{2}=0.10 ; p<0.75\right)$, separaciones af ectivas $\left(X^{2}=0.07 ; p<0.79\right)$ y despido laboral $\left(X^{2}=0.003 ; p<0.96\right)$

Respecto a las vivencias emocionales, las personas con personalidad tipo A han pasado más por períodos prolongados, mayores a los 3 meses, sintiendo tristeza $\left(X^{2}=8.01 ; p<0.05\right)$, temores $\left(X^{2}=12.57 ; p<0.01\right)$ y fatiga $\left(X^{2}=\right.$ 18.85; $\mathrm{p}<0.01)$ que las personas sin este tipo de personalidad; pero no difieren en relación a sentir intranquilidad $\left(X^{2}=5.21 ; p<0.07\right)$.

\section{Discusión}

Las personas se distinguen entre sí, tanto por sus tipos de conducta como por los efectos que éstos han tenido y tienen en su estado de salud.

Un aspecto a destacar es que la personalidad tipo A y tipo B son patrones de comportamiento extremos y es de hacer ver que tal dicotomía no es absoluta; ciertamente, la mayoría de personas se caracterizan por rasgos de ambos patrones de comportamiento (Rosenzweig, M. R. y Leiman, A. I. Op. cit.), tal como aquí se observa.

Los problemas de salud, que se asocian a los distintos tipos de conducla, también muestran importantes dilerencias en las personas: la incidencia de enfermedad cardiaca, migraña y alergias es mayor entre personas de lipo A que entre personas que no les caracteriza este patrón de comportamiento.

Las mujeres tipo A presentan cinco veces más enfermedad cardiaca y padecen cuatro veces más de migraña, que las mujeres que les distingue otro tipo de personalidad, y los hombres tipo A sufren tres veces más de alergias respecto a los hombres sin estos rasgos; en una muestra con predominancia de adultos jóvenes (entre los 21 y 40 años) y solteros.

Dos características que distinguen a las personas tipo $A$, de las personas sin este tipo de conducta, son: su mayor exposición a conflictos intrafamiliares y sus estados persistentes de tristeza, temores y/o latiga.

En síntesis, los resultados estadísticos confirman que la personalidad tipo A no sólo esta implicada en la enfermedad cardiaca, en términos concordantes con lo establecido por los resultados de los estudios referidos en el apartado introductorio de este documento, sino que también está implicada en otros trastornos psicosomáticos como la migraña y las alergias; sin embargo, los mismos resultados indican que, por la fuerza de la relación que guardan esa característica y esas condiciones, la personalidad tipo $\wedge$ no es suficiente para originarlos. 


\section{Figura 1}

\section{La reacción emocional}

Ilustración de los elementos que operan en un evento encaminado a preparar al organismo para la adaptación/ajuste

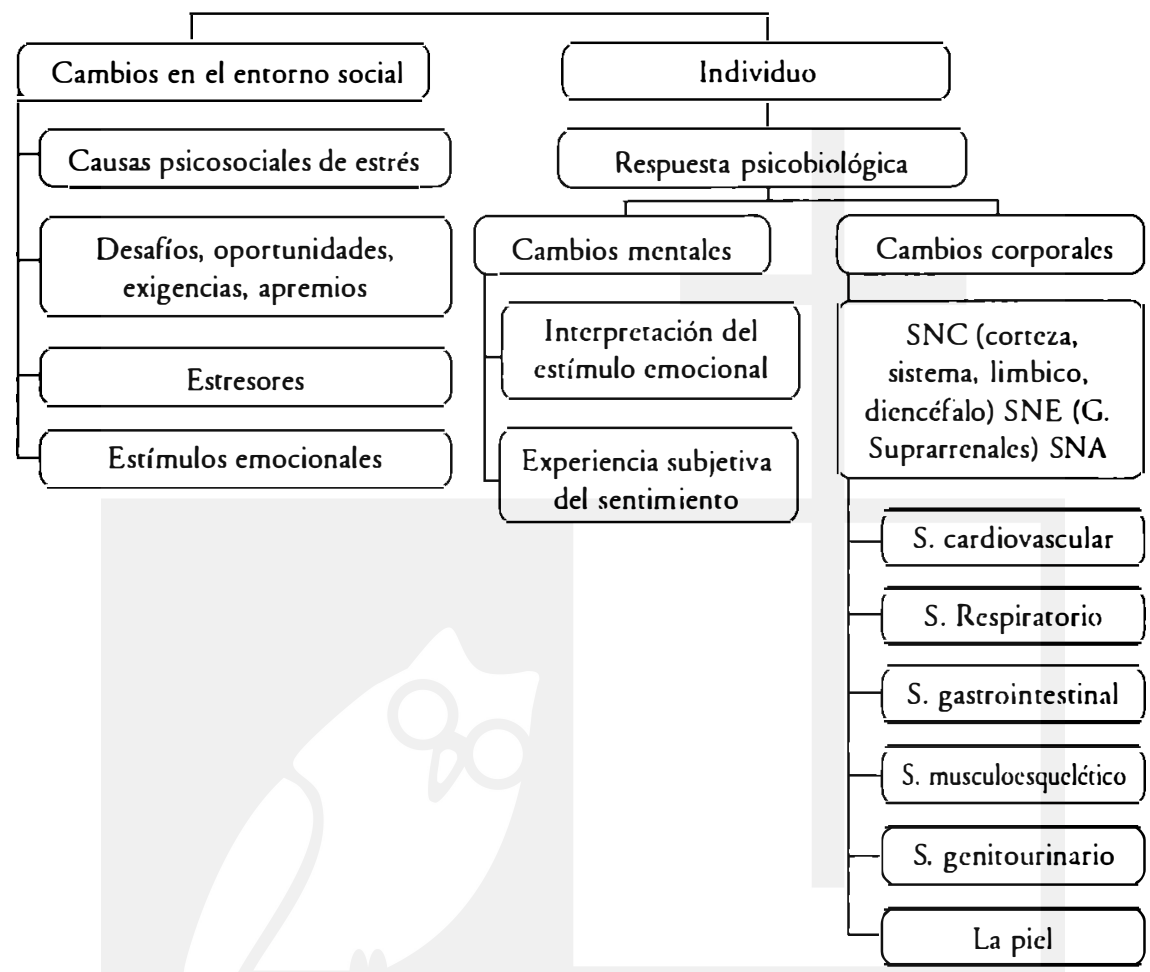

Bajo estas circunstancias, y dada la falta de una explicación oficial sobre el origen de trastornos psicosomáticos, es posible hacer un ejercicio explicativo que no sólo ubique el papel que desempeña el patrón de comportamiento en su causa, sino también, haciendo acopio de una diversidad de resultados positivos de investigación en torno a este grupo genérico de trastornos, el de otros factores igualmente contenidos en dicho fenómeno.

La emoción es una respuesta del individuo a cambios en el entorno social que implican alteraciones a su vida, por ser favorables o desfavorables; respuesta que se constituye en un proceso operativo encaminado a preparar al organismo para la adaptación o el ajuste (ver figura 1 ).

Por un lado, los cambios en el entorno social se encuentran señalados en una variedad de expresiones, tales como: desafíos, oportunidades, exigencias o apre- 
mios, limitaciones u obstáculos, causas psicosociales de estrés, estresores o estímulos emocionales.

Por otro lado, en el individuo que responde se despliegan cambios, tanto mentales como biológicos, los que, de un modo muy grueso sucederían así:

Inicialmente, una actividad cognitiva realiza la interpretación del estímulo emocional, para luego dar paso a la vivencia subjetiva del sentimiento, cuyo contenido está en correspondencia con la interpretación que se efectúa (es distinto lo que se sentirá ante un hombre con un puñal en la mano, si este es un actor que representa un papel en una obra teatral que si es un desconocido que se encuentra en la calle asaltando a una persona, justamente por la interpretación de la situación).

Simultáneamente con ello, un conjunto de sistemas orgánicos entran en actividad; a la acción cognitiva que realiza la interpretación del estímulo emocional, le subyace la actividad de diversas estructuras de la corteza cerebral, que permiten la concreción del proceso intelectivo, mientras a la vivencia del sentimiento, la de estructuras límbicas y diencefálicas, que filtran las señales y, al integrarse a la anterior, dan lugar a la elección de sus contenido. Estas desencadenan actividad endocrina, la cual incluye la secreción de adrenalina y noradrenalina por parte de la médula suprarrenal, estos productos glandulares constituyen los neurotransmisores que median los impulsos del sistema nerviosos autónomo, el cual, a través de la acción simpática, provoca la activación de los sistemas cardiovascular, respiratorio, gastrointestinal, musculoesquelético, genitourinario y la piel.

Todo esto lleva a que el individuo se vea impulsado a efectuar las manipulaciones correspondientes para asumir control sobre la situación del entomo que lo tensiona o a realizar los cambios en sí mismo que sean requeridos para amoldarse a ella; y la activación mental y biológica que configuró la respuesta del individuo cesa.

En lo dicho, hay dos puntos a resaltar: la emoción es esencialmente un evento psicobiológico de carácter reactivo, lo cual significa no sólo que aparece en la interacción del individuo con el medio físico y social, sino también que es delimitada en su inicio y terminación (de ahí que no ocurre sin razón ni es un estado permanente) y, además, es un mecanismo que opera con la finalidad de proteger la integridad psicofísica del individuo.

Sin embargo, existen factores, relativos tanto al entorno como al individuo, que pueden afectar el curso de la reacción emocional, alterando precisamente su carácter reactivo y transformándola en un estado permanente que conduce a la desadaptación o al desajuste (ver figura 2).

Los cambios en el entorno pueden suponer una tensión circunstancial o continua, esperada o indeseable, factible de dominio e incontrolable para y por parte del individuo, con una alta capacidad para afectar la respuesta emocional (como lo fue en el pasado reciente la guerra o como también lo sigue siendo hoy el desempleo, por ejemplo). 
El individuo también posee características psicológicas y biológicas que lo hacen más o menos eficaz para lidiar con las tensiones a las que se ve expuesto, lo cual depende, por una parte, de su personalidad (que abarca su tipo de conducta o patrón de comportamiento y sus capacidades apara adaptarse a los cambios del entomo, manejar y expresar sentimientos y emociones, interpretar correctamente limitaciones y oportunidades, y establecer lazos interpersonales provechosos), así como de sus experiencias (respecto a las formas de valorar las situaciones y los mecanismos de afrontamiento); y por otra parte, también depende de su vulnerabilidad orgánica y de la fuerza y patrón de respuesta fisiológica que le sea propio.

De ahí que si en estos ámbitos hay factores cuya influencia obstruya que el individuo se ajuste o adapte a la situación del entorno que lo tensiona, la activación mental y biológica que configura la respuesta del individuo persistirá y es al agotar la resistencia del organismo que ocurre el daño corporal.

Figura 2

Factores que pueden afectar el curso de la reacción emocional (su carácter reactivo), transformándolo en un estado permanente que conduce a desadaptación/desajuste

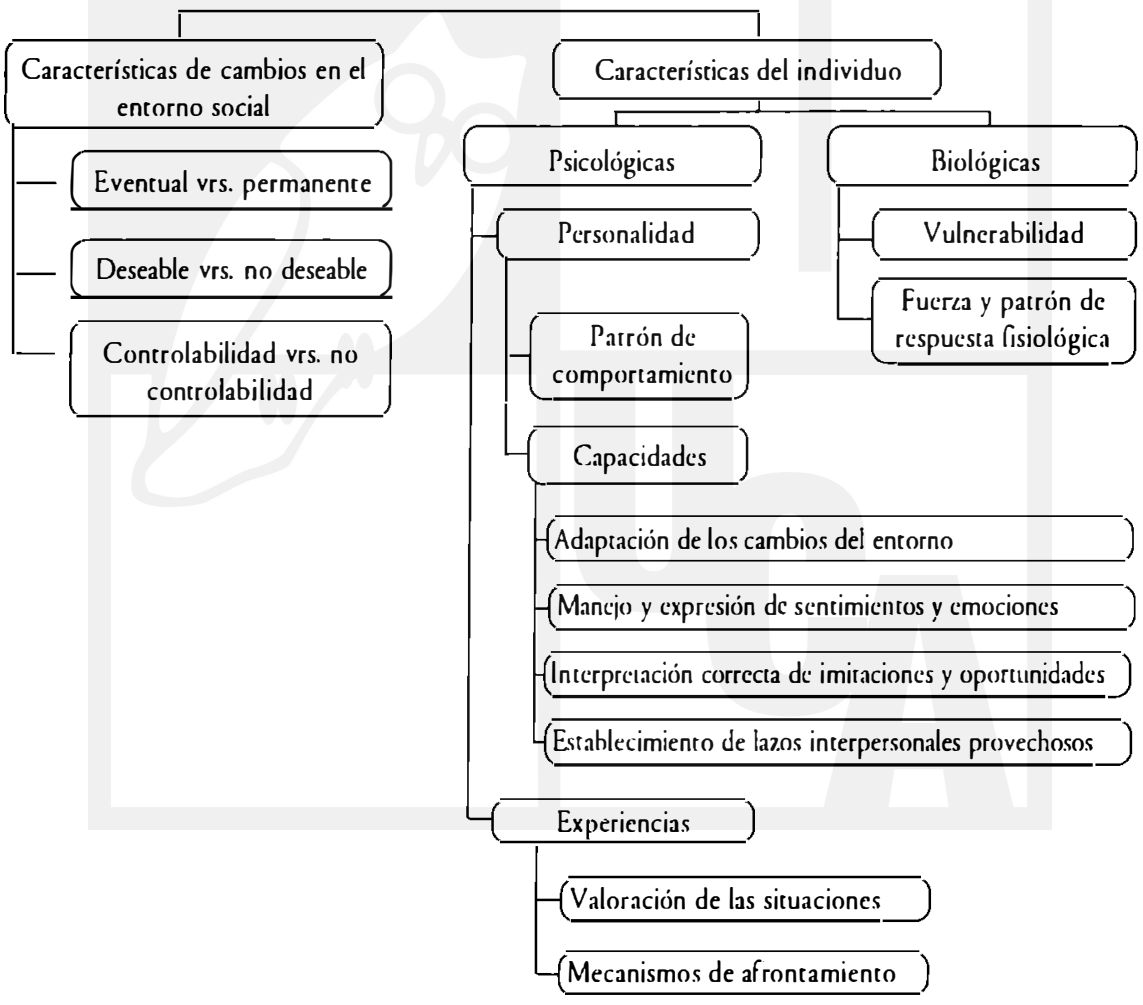


Ahora bien, si la producción del daño físico, que ocurre en el trastorno psicosomático, tiene factores sociales, psicológicos y biológicos como componentes necesarios, es sólo su concatenación lo que configura el hecho único necesario y suficiente, para originarlo; resulta obvio, entonces, que su explicación integral debe hacerse en estos términos.

Esta propuesta de explicación armoniza con la convicción, que en la actualidad está tomando cada vez más auge, de que son las interacciones de un grupo de variables (físicas, psicológicas y sociales) las que producen el fenómeno que se conoce como "enfermarse", y de que la idea de que la enfermedad se debe sólo a la influencia de agentes externos o internos es obsoleta o errónea. (Sarason, I.G. y Sarason, B.R. Op. cit.); (Selva Sutter, E. A. Epidemiologia socionatural. Su interacción con un nuevo concepto sobre la calidad de los procesos de salud-enfermedad. Mimeo., 1998.)

En un plano más disciplinar, la medicina conductual y la psicología de la salud, dos campos de reciente desarrollo, que comparten la idea de que la salud, la vulnerabilidad a la enfermedad y la reacción a la misma, están influenciadas por la combinación de factores biológicos, psicológicos y sociales, han demostrado que la mayoría de enfermedades (desde el resfriado común hasta el cáncer y las enfermedades cardiacas) pueden verse influenciadas positiva o negativamente por el estado mental, el estilo de vida y las relaciones sociales de una persona; y hacen hincapié en que la investigación encaminada a descubrir los mecanismos que yacen tras estos factores, puede indicar nuevas maneras de prevenir y tratar las enfermedades. (Sarason, I.G. y Sarason, B.R. Op. cit.)

Lo anterior sugiere que el control de los trastornos psicosomáticos requiere de la integración de campos de las ciencias biomédicas y conductuales, para el abordaje del trastorno físico y de los elementos psicológicos involucrados en su desarrollo (Nathan, P.E. y Harris, S. L. Op. cit.).

Se ha señalado que aquellos que se dedican a la evaluación y tratamiento directos del paciente que padece este tipo de trastornos deben ayudarlo a adoptar formas de vida más saludables; y que las personas que trabajan en su prevención, deben buscar reducir los riesgos para la salud, cambiando la mentalidad y los hábitos de vida de la gente o, mejor aún, tratar de fortalecer las conductas que contribuyan a la salud (Sarason, I.G. y Sarason, B.R. Op. cit.).

Lo cual, en principio, constituye un señalamiento acertado, pues hace ver la necesidad de una tarea básica; sin embargo, debe tenerse cuidado con el énfasis con que se tiende a concebir la salud como una responsabilidad individual o un logro personal, pues ya se ha planteado que factores físicos, psicológicos, sociales y económicos influyen en la salud y en la recuperación de la enfermedad; en ese sentido, no debe olvidarse que no todos los determinantes de la salud son controlables por parte del individuo y que algunos requerirán ser controlados mediante la acción social. 
Finalmente, sólo se desea hacer ver que el punto de vista aquí expuesto no sólo es relevante en la comprensión de la causalidad de la enfermedad, sino que también es importante en el establecimiento del abordaje orientado a su prevención y tratamiento.

Si creemos en ello, la tarea, ahora, sería establecer los eventos del medio y las características psicológicas y biológicas que al interactuar dan origen a cada uno de los problemas de salud antes examinados. 